\title{
WALL'S FINITENESS OBSTRUCTION
}

\author{
ERIK KJÆR PEDERSEN
}

\section{INTRODUCTION}

The purpose of this note is to give a self contained description of Wall's finiteness obstruction [8, 9]. One aim is to provide motivation for beginning students in a course on Algebraic $K$-Theory. In a final section we shall give some examples of applications and open questions relating to finiteness obstructions.

\section{Definitions and statements of theorems}

Throughout this note we work in the category of compactly generated topological spaces.

Definition 1.1. A connected topological space $X$ is finitely dominated if there exists a finite CW-complex $K$ and maps $X \stackrel{i}{\rightarrow} K$ and $K \stackrel{r}{\rightarrow} X$ so that $r \circ i$ is homotopic to the identity.

Theorem 1.2. Let $X$ be finitely dominated. Then

(1) $X$ is homotopy equivalent to a finite dimensional CW-complex.

(2) $X$ is homotopy equivalent to an infinite dimensional $\mathrm{CW}$-complex with finite skeleta.

(3) $X \times S^{1}$ is homotopy equivalent to a finite $\mathrm{CW}$-complex.

(4) There is an invariant $\sigma(X) \in \tilde{K}_{0}\left(\mathbb{Z}\left[\pi_{1}(X, x)\right]\right)$ so that $X$ is homotopy equivalent to a finite $\mathrm{CW}$-complex if and only if $\sigma(X)=0$.

(5) For every element $\sigma \in \tilde{K}_{0}\left(\mathbb{Z}\left[\pi_{1}(X, x)\right]\right)$ there exists a finitely dominated space $Y$ so that $\sigma(Y)=\sigma$.

From now on we will mostly abbreviate $\pi_{1}(X, x)$ to $\pi$. A key step in the proof is the following:

Theorem 1.3. A connected space $X$ is finitely dominated if and only if the following hold:

(1) $X$ is homotopy equivalent to a $\mathrm{CW}$-complex.

(2) $\pi_{1}(X, x)$ is finitely presented.

(3) The singular chains of the universal cover $S_{*}(\tilde{X})$ is chain homotopy equivalent as a chain complex of $\mathbb{Z}[\pi]$-modules to a finite length chain complex

$$
0 \rightarrow P_{k} \rightarrow P_{k-1} \rightarrow \ldots \rightarrow P_{0} \rightarrow 0
$$

of finitely generated projective $\mathbb{Z}[\pi]$-modules 
Remark 1.4. Assuming the theorem above we may consider

$$
\left[P_{*}\right]=\sum(-1)^{i}\left[P_{i}\right] \in K_{0}(\mathbb{Z}[\pi])
$$

By a standard argument this is a well defined invariant of the chain homotopy type of $P_{*}$, and hence a well defined invariant of $X$. The inclusion $\mathbb{Z} \rightarrow \mathbb{Z}[\pi]$ followed by the augmentation $\mathbb{Z}[\pi] \rightarrow \mathbb{Z}$ provides a splitting

$$
K_{0}(\mathbb{Z}[\pi])=K_{0}(\mathbb{Z}) \oplus \tilde{K}_{0}(\mathbb{Z}[\pi])=\mathbb{Z} \oplus \tilde{K}_{0}(\mathbb{Z}[\pi])
$$

and we may write

$$
\left[P_{*}\right]=(\chi(X), \sigma(X))
$$

It is easy to see that $\chi(X)$ is the usual Euler characteristic of $X$, and $\sigma(X)$ is now by definition the finiteness obstruction. Observe that $\sigma(X)=0$ if $X$ is homotopy equivalent to a finite $\mathrm{CW}$-complex.

To prove one direction of Theorem 1.3 we apply tricks due to M. Mather [4] and A. Ranicki [6]. Preparing for Mather's trick we use

Definition 1.5. Let $X$ be a space and $f: X \rightarrow X$ a self map. The mapping torus $T(X, f)$ is defined by

$$
T(X, f)=X \times[0,1] /(x, 1) \sim(f(x), 0)
$$

Lemma 1.6. The map $T(f): T(X, f) \rightarrow T(X, f)$ sending $(x, s)$ to $(f(x), s)$ is homotopic to the identity

Proof. A homotopy $H$ is provided by

$$
H(x, s, t)= \begin{cases}(x, s+t) & \text { if } s+t \leq 1 \\ (f(x), s+t-1) & \text { if } s+t \geq 1\end{cases}
$$

Lemma 1.7. Let $X$ and $Y$ be topological spaces and $f: X \rightarrow Y$ and $g: Y \rightarrow X$ maps. Then $T(X, g \circ f)$ and $T(Y, f \circ g)$ are homotopy equivalent spaces.

Proof. $f$ and $g$ induce maps $T(X, g \circ f) \rightarrow T(Y, f \circ g)$ and $T(Y, f \circ g)$ and $T(X, g \circ f)$, and the composites are homotopic to the identities by the lemma above.

Lemma 1.8. If $f, g: X \rightarrow X$ are homotopic maps, then $T(X, f)$ and $T(X, g)$ are homotopy equivalent.

Proof. Let $H$ be a homotopy from $f$ to $g$. Define $F: T(X, f) \rightarrow T(X, g)$ and $G: T(X, g) \rightarrow$ $T(X, f)$ by

$$
F(x, s)= \begin{cases}(x, 2 s) & \text { if } s \leq \frac{1}{2} \\ (H(x, 2 s-1), 0) & \text { if } s \geq \frac{1}{2}\end{cases}
$$




$$
G(x, s)= \begin{cases}(x, 2 s) & \text { if } s \leq \frac{1}{2} \\ (H(x, 2-2 s), 0) & \text { if } s \geq \frac{1}{2}\end{cases}
$$

It is now easy to see that $F \circ G$ and $G \circ F$ are homotopic to the respective identities by writing down explicit homotopies.

We are now ready to attack the proof of Theorem 1.3 ,

Proof of Theorem 1.3 one direction. We assume $X$ is finitely dominated so we have $X \stackrel{i}{\rightarrow} K$ and $K \stackrel{r}{\rightarrow} X$ with $r \circ i \simeq 1_{X}$. This means $T(X, r \circ i) \simeq X \times S^{1}$ is homotopy equivalent to $T(K, i \circ r)$ which is a finite $\mathrm{CW}$-complex. Now since $X \times S^{1}$ is homotopy equivalent to a CW-complex, so is the cyclic cover $X \times \mathbb{R}$ which in turn is homotopy equivalent to $X$. From now on we replace $X$ by a CW-complex. We choose a basepoint $x \in X$ and use the image as basepoint in $K$. The fundamental group of $T(K, i \circ r)$ is finitely presented since $T(K, i \circ r)$ ) is a finite $\mathrm{CW}$-complex. It follows that $\pi_{1}(X, x) \times \mathbb{Z}$ is finitely presented and by adding just one more relation $\pi_{1}(X, x)$ is finitely presented.

Now denote the map $i \circ r: K \rightarrow K$ by $p$. We know that $p \circ p$ is homotopic to $p$. We can assume that $r$ and $i$ preserve a basepoint, but we want the homotopy to preserve the basepoint as well, since this will provide us with an equivariant homotopy between $\tilde{p} \circ \tilde{p}$ and $\tilde{p}$. For this purpose we need a basepoint preserving homotopy from $r \circ i$ to $1_{X}$. We now choose a special domination namely $X \rightarrow X \times S^{1} \simeq L$ with the obvious retraction. The homotopy equivalence $X \times S^{1} \rightarrow L$ induces isomorphism of homotopy groups, and hence, since it is a map of CW-complexes, we may use Whitehead's theorem to produce a pointed homotopy equivalence inducing the given isomorphism on homotopy groups. This produces a pointed homotopy between $p$ and $p \circ p$ and hence an equivariant homotopy between $\tilde{p}$ and $\tilde{p} \circ \tilde{p}$. It is convenient to have the domination induce isomorphism on the fundamental group, but this may be obtained by adding a 2-cell to $L$.

Now consider the singular functor sending $[n]$ to

$$
S_{n}(\tilde{X})=\left\{\sigma: \Delta^{n} \rightarrow \tilde{X}\right\}
$$

followed by realization

$$
|S .(\tilde{X})|=\coprod_{n} S_{n}(\tilde{X}) \times \Delta^{n} /\left\{\alpha_{*} \sigma(x) \simeq \sigma\left(\alpha^{*}(x)\right)\right\}
$$

Evaluation gives a continuous map $\left|S_{*}(\tilde{X})\right| \rightarrow \tilde{X}$ which is $\pi$-equivariant. We approximate to a cellular map $\pi$-equivariantly and apply cellular chains on both sides. The cellular chains on the left side is the same as the (normalized) singular chain complex and the map induces an isomorphism in homology. Since we have chain complexes of free $\mathbb{Z}[\pi]$-modules, the chain complexes are chain homotopy equivalent. The proof of this direction is now finished by applying the following lemma due to A. Ranicki [6]. 
Lemma 1.9. Let $R$ be a ring and $A_{*}$ be a chain complex. Assume there exists a finite length chain complex of finitely generated projective $R$-modules $C_{*}$, and chain maps $i: A_{*} \rightarrow C_{*}$ and $r: C_{*} \rightarrow A_{*}$ so that $r \circ i$ is chain homotopic to the identity. Then $A_{*}$ is homotopy equivalent to an infinite length chain complex of finitely generated projective modules

$$
0 \rightarrow F_{n} \rightarrow F_{n-1} \rightarrow \cdots \rightarrow F_{1} \rightarrow F \stackrel{P}{\rightarrow} F \stackrel{1-P}{\rightarrow} F \stackrel{P}{\rightarrow} F \stackrel{1-P}{\rightarrow} \cdots
$$

where $P: F \rightarrow F$ is a projection.

Remark 1.10. It is easy to see this chain complex in turn is chain homotopy equivalent to

$$
0 \rightarrow F_{n} \rightarrow F_{n-1} \rightarrow \cdots \rightarrow F_{1} \rightarrow \operatorname{ker}(P) \rightarrow 0
$$

which is what we have been aiming for. Notice that if the modules $C_{i}$ are free we get an infinite chain complex of free modules, but in the end we still only get a finite length chain complex of projective modules.

Proof of lemma. Let $C_{*}: 0 \rightarrow C_{n} \rightarrow \cdots \rightarrow C_{1} \rightarrow C_{0} \rightarrow 0$ be a dominating complex so we have $A_{*} \stackrel{i}{\rightarrow} C_{*}$ and $C_{*} \stackrel{r}{\rightarrow} A_{*}$ and a chain homotopy $s$ of $A_{*}$ so $s \partial+\partial s=1-r \circ i$. Define $F=C_{0} \oplus C_{1} \oplus \cdots \oplus C_{n}$. Then

$$
P=\left(\begin{array}{cccccc}
i r & \partial & 0 & 0 & 0 & \ldots \\
i s r & 1-i r & -\partial & 0 & 0 & \ldots \\
i s^{2} r & -i s r & i r & \partial & 0 & \ldots \\
i s^{3} r & -i s^{2} r & i s r & 1-i r & -\partial & \ldots \\
i s^{4} r & -i s^{3} r & i s^{2} r & -i s r & i r & \ldots \\
\vdots & \vdots & \vdots & \vdots & \vdots &
\end{array}\right)
$$

has $P^{2}=P$. Defining

$$
F_{r}=C_{r} \oplus C_{r+1} \oplus \cdots \oplus C_{n}
$$

and $\partial: F_{i} \rightarrow F_{i-1}$ by

$$
\left(\begin{array}{cccc}
\partial & 0 & 0 & \cdots \\
1-i r & -\partial & 0 & \cdots \\
-i s r & i r & \partial & \ldots \\
i s^{2} r & i s r & 1-i r & \ldots \\
\vdots & \vdots & \vdots &
\end{array}\right)
$$

we get a chain complex $F_{*} \quad 0 \rightarrow F_{n} \rightarrow \cdots \rightarrow F_{1} \rightarrow F \stackrel{P}{\rightarrow} F \stackrel{1-P}{\rightarrow} F \cdots$ which one may check is homotopy equivalent to $A_{*}$ by

$$
I: A_{*} \rightarrow F_{*} \quad \text { and } \quad R: F_{*} \rightarrow A_{*}
$$


WALL'S FINITENESS OBSTRUCTION

where

$$
I=\left(\begin{array}{c}
i \\
i s \\
i s^{2} \\
\vdots
\end{array}\right): A_{m} \rightarrow F_{m}
$$

and $R=(r, 0,0, \ldots): F_{m} \rightarrow A_{m}$. Since $R \circ I=r \circ i \sim 1_{A}$ and the projection $F_{r}=$ $C_{r} \oplus C_{r-1} \oplus \cdots \oplus C_{n} \rightarrow F_{r+1}=C_{r+1} \oplus \cdots \oplus C_{n}$ is a homotopy from $I R$ to the identity, we are done.

We now consider the proof of the other direction of Theorem 1.3. For this we need some lemmas.

Lemma 1.11. Given a pair of topological spaces $(X, Y)$ with $\pi_{1}(X, y) \cong \pi_{1}(Y, y)=\pi$ and a collection of elements $a_{i} \in \pi_{k}(X, Y), k \geq 2$ represented by $\alpha_{i}:\left(D^{k}, S^{k-1}\right) \rightarrow(X, Y)$. Let $Z=\bigcup_{i} Y \cup_{\alpha_{i}} D^{k}$ and extend the map from $Y \rightarrow X$ to $Z \rightarrow X$ using $\alpha_{i}$. Then $H_{l}(\tilde{X}, \tilde{Z}) \cong$ $H_{l}(\tilde{X}, \tilde{Y})$ for $l \neq k, k+1, H_{k}(\tilde{Z}, \tilde{Y})=\bigoplus_{i} \mathbb{Z}[\pi]$ and we have an exact sequence

$$
0 \rightarrow H_{k+1}(\tilde{X}, \tilde{Y}) \rightarrow H_{k+1}(\tilde{X}, \tilde{Z}) \rightarrow H_{k}(\tilde{Z}, \tilde{Y}) \rightarrow H_{k}(\tilde{X}, \tilde{Y}) \rightarrow H_{k}(\tilde{X}, \tilde{Z}) \rightarrow 0
$$

and the map

$$
\oplus_{i} \mathbb{Z}[\pi] \cong H_{k}(\tilde{Z}, \tilde{Y}) \rightarrow H_{k}(\tilde{X}, \tilde{Y})
$$

is given by $\pi_{k}(X, Y, x) \cong \pi_{k}(\tilde{X}, \tilde{Y}, \tilde{x})$ followed by the Hurewicz homomorphism applied to the $\alpha_{i}$.

Notice that if $k=2$, we have $\alpha_{i}: S^{1} \rightarrow Y$ is null homotopic since we have isomorphic fundamental groups.

Proof. Use the long exact sequence of the triple $(\tilde{Z}, \tilde{Y}) \rightarrow(\tilde{X}, \tilde{Y}) \rightarrow(\tilde{X}, \tilde{Z})$ and excision on the pair $(\tilde{Z}, \tilde{Y})$.

Lemma 1.12. Let $0 \rightarrow P_{n} \rightarrow P_{n-1} \rightarrow \ldots \rightarrow P_{2} \rightarrow P_{1} \rightarrow P_{0} \rightarrow 0$ be a chain complex of finitely generated projective $R$-modules. Assume $H_{i}\left(P_{*}\right)=0$ for $i \leq k$. Then $P_{*}$ is chain homotopy equivalent to a chain complex of finitely generated projective $R$-modules of the form $0 \rightarrow P_{n} \rightarrow \ldots P_{k+2} \rightarrow Q \rightarrow 0$. In particular $H_{k+1}\left(P_{*}\right)$ is finitely generated.

Proof. Assuming $k \geq 0$ we have $P_{1} \rightarrow P_{0}$ is onto and by projectivity we may split the map and write $P_{1}=P_{0} \oplus Q_{1}$ and the map projection on the first summand. If $k>0$ we may repeat this argument until we meet a non-vanishing homology group.

We are now ready for

Proof of Theorem 1.3 other direction. Since $\pi_{1}(X, x)$ is finitely presented we can construct a connected pointed 2-complex $K$ and a pointed map $K \rightarrow X$ inducing isomorphism on $\pi_{1}$. The Hurewicz theorem shows that $\pi_{2}(\tilde{K}, \tilde{k}) \rightarrow H_{2}(\tilde{K})$ and $\pi_{2}(\tilde{X}, \tilde{x}) \rightarrow H_{2}(\tilde{X})$ are isomorphisms. It now follows from the five lemma that $\pi_{2}(\tilde{X}, \tilde{K}, \tilde{k})=H_{2}(\tilde{X}, \tilde{K})$. It follows 
from the lemmas above that this is a finitely generated $\mathbb{Z}[\pi]$-module since it is the homology of the mapping cone of $C_{*}(\tilde{K}) \rightarrow P_{*}$, a finite length chain complex of finitely generated projective modules. Choose a generating set $\alpha_{i}:\left(D^{2}, S^{1}\right) \rightarrow(X, K)$ and attach cells to obtain a space $L$, and extend the map. It follows from the lemma above that $H_{i}(\tilde{X}, \tilde{L})=0$ for $i \leq 2$. We now continue using the relative Hurewicz theorem to attach cells to obtain a space we shall again denote as $L$ so that $H_{i}(\tilde{X}, \tilde{L})=0$ for $i \neq k$ and $H_{k}(\tilde{X}, \tilde{L})=P$ a projective $\mathbb{Z}[\pi]$-module. Now consider $\left(\widehat{X \times S^{1}}, \widehat{L \times S^{1}}\right)$. We have not changed the homology since the universal cover of $S^{1}$ is contractible. Choose a projective module $Q$ so that $P \oplus Q$ is free and consider the short exact sequence

$$
0 \rightarrow P \otimes \mathbb{Z}\left[t, t^{-1}\right] \oplus Q \otimes \mathbb{Z}\left[t, t^{-1}\right] \stackrel{(1-t) \oplus 1}{\longrightarrow} P \otimes \mathbb{Z}\left[t, t^{-1}\right] \oplus Q \otimes \mathbb{Z}\left[t, t^{-1}\right] \rightarrow P \rightarrow 0
$$

The two first terms are finitely generated free $\mathbb{Z}[\pi]\left[t, t^{-1}\right]$-modules, so we may first attach $k$ cells to $L \times S^{1}$ and then $(k+1)$-cells to obtain a finite complex which is homotopy equivalent to $X \times S^{1}$ since the relative homology of the universal cover is trivial. This shows that $X$ is finitely dominated namely by a finite complex homotopy equivalent to $X \times S^{1}$

We now turn to the proof of theorem 1.2

Proof of Theorem 1.2. We continue the argument from the proof above and assume we have constructed a finite complex $L$ and a map $L \rightarrow X$ so that $\pi_{1}(L, l) \rightarrow \pi_{1}(X, x)$ is an isomorphism and $H_{l}(\tilde{X}, \tilde{L})$ is 0 for $l \neq k$ and $H_{k}(\tilde{X}, \tilde{L})$ is a finitely generated projective $\mathbb{Z}[\pi]$-module which we denote by $P$. Choose a finitely generated projective module $Q$ so that $P \oplus Q$ is free. Consider the short exact sequence

$$
0 \rightarrow Q \oplus P \oplus Q \oplus P \oplus Q \ldots \rightarrow P \oplus Q \oplus P \oplus Q \oplus P \ldots \rightarrow P \rightarrow 0
$$

where the map is given by shifting by one. The first two terms are free and we can use a basis to attach infinitely many $k$-cells corresponding to the middle term, and then infinitely many $(k+1)$-cells corresponding to the first term to obtain a finite dimensional $\mathrm{CW}$-complex so the relative homology of the universal cover is 0 hence $X$ is homotopy equivalent to a finite dimensional CW-complex.

To see $X$ is homotopy equivalent to an infinite dimensional CW-complex with finite skeleta consider the short exact sequence

$$
0 \rightarrow Q \rightarrow P \oplus Q \rightarrow P \rightarrow 0
$$

The middle term is free, so we may attach $k$-cells to obtain a complex so the relative homology of the universal cover is $Q$ in dimension $k+1$. Then consider the short exact sequence

$$
0 \rightarrow P \rightarrow P \oplus Q \rightarrow Q \rightarrow 0
$$

and attach $(k+1)$-cells. Continuing this process ad infinitum we only attach finitely many cells in each dimension to obtain an infinite dimensional complex with the relative homology of universal covers 0 , hence homotopy equivalent to $X$. 
We have already shown $X \times S^{1}$ is homotopy equivalent to a finite $\mathrm{CW}$-complex using Mather's trick, and we have shown it again above if we used the alternative definition of finitely dominated suggested by theorem 1.3. The element $0 \in \tilde{K}_{0}(\mathbb{Z}[\pi])$ is represented by a finitely generated free module so if $X$ is homotopy equivalent to a finite complex $K$ we see that $S_{*}(\tilde{X})$ is homotopy equivalent to the cellular chains of $\tilde{K}$ and hence $\sigma(X)=0 \in$ $\tilde{K}_{0}(\mathbb{Z}[\pi])$.

Now we need to show that if $\sigma(X)=0$ then $X$ is homotopy equivalent to a finite complex. Constructing $L$ as above it is easy to see that $\sigma(X)= \pm[P]$ according to whether $k$ is even or odd. If $\sigma(X)=0$ this means that $P$ is stably free, so we may construct a short exact sequence

$$
0 \rightarrow E \rightarrow F \rightarrow P \rightarrow 0
$$

where $E$ and $F$ are finitely generated and free. We now attach finitely many $k$-cells corresponding to generators of $E$ and finitely many $(k+1)$-cells corresponding to generators of $F$ to obtain a finite complex homotopy equivalent to $X$.

Finally let us show that for every element $\sigma \in \tilde{K}_{0}(\mathbb{Z}[\pi])$ there exists a finitely dominated space $Y$ with $\sigma(Y)=\sigma$. We may represent $\sigma$ by a finitely generated projective module $P$, and choose a finitely generated $Q$ so that $P \oplus Q$ is finitely generated free. Now choose a finite complex $K$ with $\pi_{1}(K, k)=\pi$. Let $L$ be $K$ wedge a countably infinite wedge of $k$

spheres $k \geq 2$. We now have $H_{*}(\tilde{L}, \tilde{K})$ is 0 except for $*=k$ and we may identify $H_{k}(\tilde{L}, \tilde{K})$ with an infinite sum of $Q \oplus P$. Use the short exact sequence

$$
0 \rightarrow Q \oplus P \oplus Q \ldots \rightarrow P \oplus Q \oplus P \oplus Q \ldots \rightarrow P \rightarrow 0
$$

to attach $(k+1)$-cells to $L$ to obtain $Y$ such that $H_{*}(\tilde{Y}, \tilde{K})$ is 0 except for one dimension where it is $P$. Crossing with $S^{1}$ we may argue as above to show $Y \times S^{1}$ is homotopy equivalent to a finite complex, hence $Y$ is finitely dominated, and clearly $\sigma(Y)=\sigma$.

This ends the proof of Theorem 1.2.

\section{EXAMPles AND CONJECTURES}

It is natural to consider which finiteness obstructions may be realized for various classes of spaces. Here we give some examples of that and some conjectures. Recall an $H$-space is a pointed space $X$ together with a map $X \times X \rightarrow X$ so that the composite

$$
X \vee X \subset X \times X \rightarrow X
$$

is homotopic to the folding map. Examples are topological groups and loop spaces.

Conjecture 2.1. Let $X$ be a finitely dominated $H$-space, then $\sigma(X)=0$.

For $H$-spaces that are CW-complexes G. Mislin [5] has shown that $X$ is finitely dominated if and only if $\oplus H_{i}(X, \mathbb{Z})$ is a finitely generated abelian group. The conjecture above has been verified in a number of cases.

Proposition 2.2. If $\pi_{1}(X, x)$ is infinite, then $\sigma(X)=0$. 
Proof. Being an $H$-space $\pi_{1}(X, x)$ is abelian, so being infinite $\pi_{1}(X, x) \cong \mathbb{Z} \oplus A$. Now let $S^{1} \rightarrow X$ represent a generator of $\mathbb{Z}$ and use the projection $\mathbb{Z} \oplus A \rightarrow \mathbb{Z}$ to define an element in $H^{1}(X, \mathbb{Z})$ which in turn may be represented by a map $X \rightarrow S^{1}$. The composite $S^{1} \rightarrow X \rightarrow S^{1}$ is homotopic to the identity. Let $Y$ be the homotopy fibre of $X \rightarrow S^{1}$ and consider

$$
Y \times S^{1} \rightarrow X \times X \rightarrow X
$$

This induces an isomorphism of homotopy groups so $X \simeq Y \times S^{1}$. We clearly have $Y$ is finitely dominated so $\sigma(X)=0$ by Theorem 1.2 .

Another case where the conjecture has been verified is when $X$ has a classifying space i. e. $X \simeq \Omega B$ [1.

Theorem 2.3. If $X \simeq \Omega B$ for some $\mathrm{CW}$-complex $B$, and $\oplus H_{i}(X, \mathbb{Z})$ is a finitely generated abelian group, then $X$ is homotopy equivalent to a smooth, compact, parallellizable manifold.

The first step in the proof of this theorem is to deal with the finiteness obstruction.

Another example is finitely dominated nilpotent spaces [2]. In this case there is a group between 0 and $\tilde{K}_{0}(\mathbb{Z}[\pi])$ which describes precisely which elements can be realized by a nilpotent space with fundamental group $\pi$. There are examples where this group is strictly bigger than 0 and strictly smaller than $\tilde{K}_{0}(\mathbb{Z}[\pi])$.

Another conjecture concerns classifying spaces of groups.

Conjecture 2.4. Let $\Gamma$ be a group, $B \Gamma$ its classifying space. Assume $B \Gamma$ is finitely dominated, then $\sigma(B \Gamma)=0$

This conjecture has also been verified in many cases since it has been proved in many cases that $\tilde{K}_{0}(\mathbb{Z}[\Gamma])=0$. This is a consequence of the Hsiang conjecture, see W.-C. Hsiang [3], which says that the assembly map

$$
B \Gamma_{+} \wedge K(\mathbb{Z}) \rightarrow K(\mathbb{Z}[\Gamma])
$$

is a homotopy equivalence of spectra for $\Gamma$ torsion free. Here $K(-)$ is non-connective $K$ theory. The Hsiang conjecture has been verified in a large number of cases by Farrell, Jones, Lück, Bartels, Reich, and others.

Finally it is natural to mention the first case where the finiteness obstruction occurred at least morally, namely in R. Swan's work [7] on CW-complexes with universal cover homotopy equivalent to a sphere. Here Swan constructed many examples that were finitely dominated, but with non-trivial finiteness obstruction.

\section{REFERENCES}

[1] Tilman Bauer, Nitu Kitchloo, Dietrich Notbohm, and Erik Kjær Pedersen, Finite loop spaces are manifolds, Acta Math. 192 (2004), 5-31.

[2] J. Ewing, P. Löffler, and E. K. Pedersen, A local approach to the finiteness obstruction, Quart. J. Math. Oxford Ser. (2) 39 (1988), 443-462. 
[3] W. C. Hsiang, Geometric applications of Algebraic K-theory, Proc. I. C. M. 1983, Warsaw, North Holland, 1984, pp. 99-118.

[4] M. Mather, Counting homotopy types of topological manifolds, Topology 4 (1965), 93-94.

[5] G. Mislin, Finitely dominated nilpotent spaces, Ann. of Math. (2) 103 (1976), 547-556.

[6] A. A. Ranicki, The algebraic theory of finiteness obstructions, Math. Scand. 57 (1985), 105-126.

[7] R. Swan, Periodic resolutions for finite groups, Ann. of Math. (2) 72 (1960), 267-291.

[8] C. T. C. Wall, Finiteness conditions for CW-complexes, Ann. of Math. (2) 81 (1965), 56-69.

[9] _ Finiteness conditions for CW-complexes II, Proc. Roy. Soc. London Ser. A 295 (1966), 129-139.

Department of Mathematical Sciences, University of Copenhagen, Denmark 